\title{
Invited Tutorial:
}

\section{Verification of Infinite-State and Parameterized Systems}

\author{
Parosh Aziz Abdulla and Bengt Jonsson \\ Department of Computer Systems \\ Uppsala University \\ Uppsala, Sweden
}

\begin{abstract}
Over the last few years there has been an increasing research effort directed towards the automatic verification of infinite-state systems. There are now verification techniques for many classes of infinitestate systems, including timed and hybrid automata, petri nets, pushdown systems, systems with FIFO channels, systems with a simple treatment of data, etc. In this tutorial, we will cover general verification techniques that have been used for infinite-state and parameterized systems, and try to show their power and limitations. Such techniques are e.g., symbolic model-checking techniques, abstraction, induction over the networks structure, widening, and automata-based techniques. We will focus on linear-time safety and liveness properties.
\end{abstract}

